\title{
Frequency Estimation for Short
}

\section{Realization of Radar Signals}

\author{
II. Results of Tests
}

\author{
Mikhail Ronkin \\ Department of Radioelectronic and information systems \\ Ural Federal University \\ 620002 Mira 32, Ekaterinburg, Russia \\ Ekaterina Khrestina \\ Department of Radioelectronic and information systems \\ Ural Federal University \\ 620002 Mira 32, Ekaterinburg, Russia \\ Aleksey Kalmikov \\ Department of Radioelectronic and information systems \\ Ural Federal University \\ 620002 Mira 32, Ekaterinburg, Russia
}

Copyright (C) 2014 Mikhail Ronkin, Ekaterina Khrestina and Aleksey Kalmikov. This is an open access article distributed under the Creative Commons Attribution License, which permits unrestricted use, distribution, and reproduction in any medium, provided the original work is properly cited.

\begin{abstract}
The paper is devoted to the problem of short time delays measurement in radar systems. The algorithm which was proposed by authors in paper I [1] it compared with the generally used method based on fast Fourier transform. In numerical simulation estimation of random error are carried out depend on value of delay and value of signal to noise ratio.
\end{abstract}


Keywords: radars, chirp, signal processing, frequency estimation

\section{Introduction}

The first part of paper proposed new algorithm for measurement of short time delays for linearly frequency modulated signals. That actual for many short range radar and ultrasonic measurements with linear frequency modulated (chirp) signals [1].

The principle of designed algorithm involves heterodyne processing of received chirp signal; quadrature decomposition of obtained beating signal. Than relation between phases changing and time are carried out in digital form. Such relation has leaner form in case without noises. In practice some level of white noises always exist, that's why the linearization has been performed by least squares method. Frequency of beating signal has been estimated by computation the slope angel of obtained relation. The time of delay computed from the relation with the estimated frequency [1].

It was noted in paper I [1] that Fast Fourier Transform (FFT) based methods are commonly used for frequency estimation. However those algorithms require at least 2-3 period of processed signal [2]. Such conditions are unacceptable in some short range radar applications. For decreasing the minimum measured value of time the described algorithm has been proposed. The second part of the article present results of comparison between value of randomly component of error for proposed and FFT-based algorithms depend on number of periods in time of observation and depend on signal to noise ratio.

\section{Main part}

In program «MATLAB» the scheme which was proposal in part 1 simulated for examination of proposed method. The main aim of simulation was comparing between developed method and general used algorithm of estimation by determination of maximum position in Fourier spectra. Comparison was carried out depending on signal to noise ration and depending on time of delay. Beating signal digitized with frequency in 100 times more than estimated. Approximately value of delay may be computed from expected distance to the aim. The Nyquist to beating frequency ratio is heuristically optimal. If ratio was lower error would be increase. If ratio was higher error would be either rise or without change. It can be in general explained on the one hand by reduction number of samples with decreasing Nyquist frequency. One the other hand by broadening of the frequency bandwidth with increasing sampling rate and hence rising of the power of white noise contained in it. Further detailed study of this issue is left beyond the Article. Furthermore it should be noted about feasibility of the requirement of the Nyquist to heterodyne frequency ration. Since the beating signals generally has low value 
of frequency (about 1-10 kHz), that's why discretization can be carried out on ADC with sampling rate 1-10 Mhz. Such devices are wildly available and industrially produced.

The numerical experiments have been consisted from measurements of the standard deviation (STD) of estimated frequency in relation with the time of delay (number of periods in time of modulation $(\mathrm{N})$, which equal with time of observation) and in relation with the signal to noise ratio (SNR).

The estimation of beating frequency has been performed by proposed algorithm (pr.alg.) and by determination of peak position in spectral domain. In second method specters have been obtained by FFT with extending length of samples by zeros in 300 times. Such extension are necessary for obtain high resolution without any interpolations. Quadrature decomposition has been carried out by the time domain Hilbert transform. Each value of STD is result of 100 cycles of simulations.

On the figures 1, 2 have been illustrated plots of STD normalized on beating frequency depend on number of periods in time of observation. The relations on plots shown for signal to noise ratio $3,10,20,50 \mathrm{~dB}$.

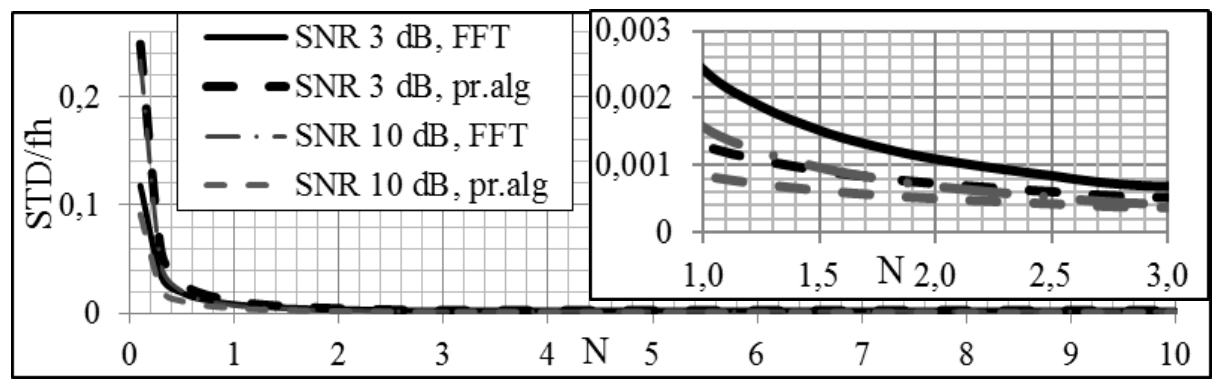

Figure 1 - STD normalized on beating frequency depend on number of periods in time of observation (N) with SNR 3 and $10 \mathrm{~dB}$.

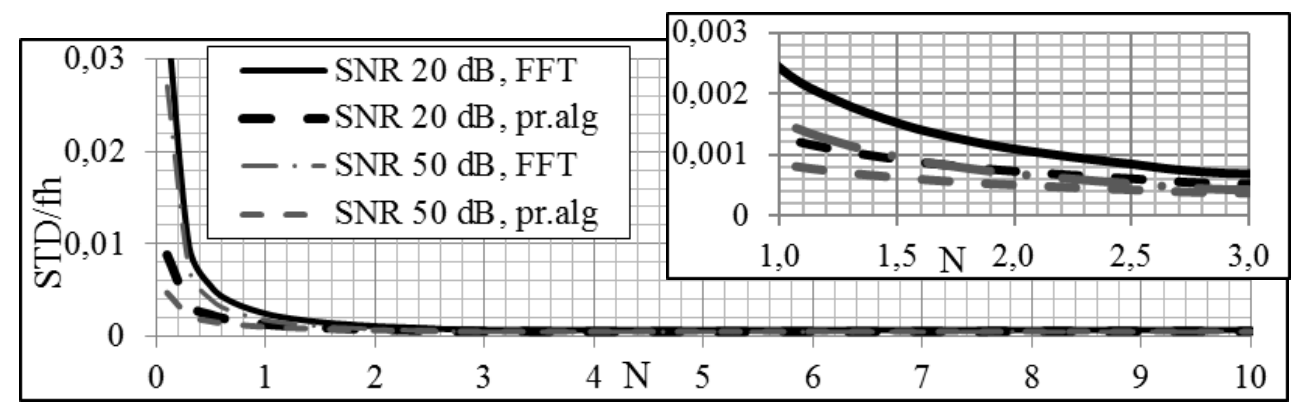

Figure 2 - STD normalized on heterodyne frequency depend on number of periods in time of observation (N) with SNR 20 and $50 \mathrm{~dB}$.

It can be noted on the figures 1, 2 that if SNR more than $10 \mathrm{~dB}$ proposed algorithm provide higher accuracy than generally used FFT-based method, especially in case of 
realization shorter than 2 period of beating signal. It can be explained by increasing importance of discrete error and by broadening of peak in spectra domain in FFT based method. The proposed algorithm based on different with FFT principle. In designed method a priori information about linearity relation of phase from time has been used. When SNR less than $3 \mathrm{~dB}$ FFT basic method has better result that can be explained by insufficiently of simple least squares method.

When length of samples more than two periods of beating signal the accuracy of measurement become dependent only from SNR ha agreement with basic theory [3]. The dependence of STD from SNR is shown on figure 3.

As it shown at the figure 3 when signal to noise ratio higher than $40 \mathrm{~dB}$ the error is relatively constant. In this case it can be noted, as it was mentioned above, the error depends on the number of period in time of observation $(\mathrm{N})$, especially when $\mathrm{N}$ less than 1, 5 periods. For instance when $\mathrm{N}$ is changing from 0,5 to 1,5 value of error increase in 5 times. In case of SNR less than $40 \mathrm{~dB}$ value of error has been meaningfully changed. In range from 40 to $10 \mathrm{~dB}$ it is changing was 3-5 times. The difference between proposed algorithm and FFT-based method decreased with rising of SNR. Except on the random component of error for the realization less than 1 period the constant component of error are significant too. Such kind of error can be explained by extension of the peak in spectral domain, and by the Gibbs phenomena [4].

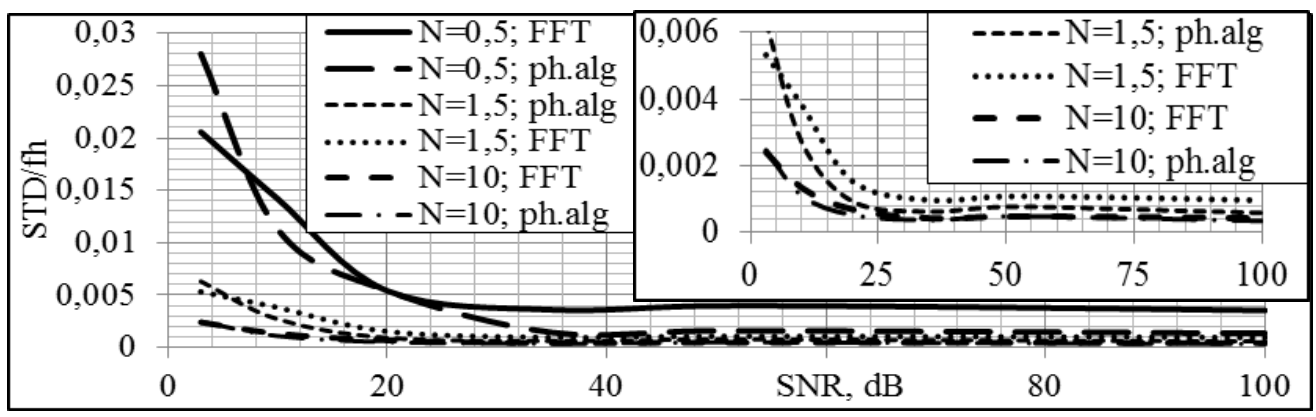

Figure 3 - STD normalized on beating frequency depend on SNR for simulation with number of periods in time of observation $0,5,1,5$ and $10 \mathrm{~N}$

It can be expected that in experimental continuation of this work such kind of error will be eliminated by using analog type of quadrature decomposition.

\section{Conclusion}

The simulation comparison of algorithm which was proposed by authors with traditionally used FFT-based method is carried out.

In the numerical experiments estimation of randomization error depends on time of observation and signal to noise ratio was performed. Proposed algorithm has 
been shown significantly benefits in case of signal to noise ratio more than $10 \mathrm{db}$. In particular advantages have been appeared when number of period in time of observation less than two.

In the paper the issues about optimal number of samples in realization and about elimination of continuous error when realizations less than 1 period left opened.

Acknowledgements. This research project has been supported by UrFU under the Framework Programme of development of UrFU through the «Young scientists UrFU» competition.

\section{References}

[1] Ronkin M., Khrestina E. Kalmykov A., Frequency estimation for short realization of radar signals. I. The new algorithm presentation, Contemporary engineering Sciences [In Print], 2014.

[2] Antipov S. A., Gnezdilov D. S., Kozmin V. A. and Stopkin V. M. Digital interpolation algorithms for estimating the frequency of the harmonic signal. Comparative analysis, Radiotekhnika, 3, (2014), 42 - 46.

[3] Van Trees H. Detection, estimation and modulation theory, Wiley, New York, 1971.

[4] Oppenheimer A., Sharf R. Discrete-time signal processing, Prentice Hall, New Jersey, 1999.

Received: October 29, 2014; Published: December 4, 2014 